\title{
PAIRS OF RECTILINEAR CONGRUENCES WITH GENERATORS IN ONE-TO-ONE CORRESPONDENCE*
}

BY

\author{
ALEXANDER J. COOK
}

\section{INTRODUCTION}

The place which the configuration composed of a pair of rectilinear congruences with generators in one-to-one correspondence occupies in differential geometry is of some prominence. When corresponding generators do not intersect, a general theory for the projective study of the configuration may be constructed from a system of linear partial homogeneous differential equations of the first order in four dependent and two independent variables.

The theory seems to be well adapted for the study of certain correspondences of Fubini, and a complete basis is furnished for their further investigation. The relation of Fubini's work to the earlier work of Darboux is also fully established. In connection with these correspondences a generalization to surfaces is secured for the concept of intersector curve which Lane introduced in his study of pairs of ruled surfaces.

The consideration of corresponding nets of curves on the intersector surfaces suggests itself, and when the nets of curves are conjugate nets on the surfaces, two distinct possibilities occur. The first of these has been studied by Fubini, and the properties which he discovered are readily obtained by the methods of the present theory. In addition, one or two new theorems are discovered. The second case arises when a family of intersector surfaces exists in association with each of the two congruences in correspondence.

\section{The DIFfERENTIAL EQUATIONS}

Let

$$
y_{k}^{i}=y_{k}^{i}(u, v) \quad(k=1,2,3,4)
$$

be the equations of four $\dagger$ surfaces $S_{i}(i=1, \cdots, 4)$. When $u=u_{0}, v=v_{0}$, four points $P_{i}$ are obtained, one for each surface. Let $l_{1}$ be the line joining $P_{1}, P_{2}$, and $l_{2}$ the join of $P_{3}, P_{4}$. As $u$ and $v$ take on all admissible values,

\footnotetext{
* Presented to the Society, June 20, 1929; received by the editors in April, 1929.

$\dagger$ The subscript or superscript letters $h, i, j, k$ will be taken to mean $(1,2,3,4)$ throughout the discussion.
} 
$l_{1}$ generates a rectilinear congruence $\Gamma_{1}$ as long as $S_{1}$ and $S_{2}$ do not degenerate into fixed points, into curves whose parameters are dependent, or finally into a fixed point and a curve respectively. Likewise the lines $l_{2}$ ordinarily determine a congruence $\Gamma_{2}$, whose generators are in one-to-one correspondence with the generators of $\Gamma_{1}$.

It will be assumed in what follows that $l_{1}$ and $l_{2}$ are skew lines, so that

$$
\Delta=\left|y^{1} y^{2} y^{3} y^{4}\right| \neq 0 \text {. }
$$

A system of linear differential equations of the first order may therefore be constructed of the form

$$
y_{u}^{i}=a^{i j} y^{j}, \quad y_{v}^{i}=b^{i j} y^{j},
$$

where each $y^{i}$ stands for $y_{k}{ }^{i}$, and where the summation convention used in tensor analysis has been introduced.

Suppose now that we are presented with a system of equations in four dependent variables $y^{i}$ and two independent variables $u$ and $v$ of the form (2.1). The system will be completely integrable when and only when the conditions

$$
a_{v}^{i j}+a^{i k} b^{k j}=b_{u}^{i j}+b^{i k} a^{k j}
$$

are satisfied. In particular we find

$$
\sum_{i=1}^{4} a_{v}^{i i}=\sum_{i=1}^{4} b_{u}^{i i} .
$$

Hence $f(u, v)$ exists such that

and further

$$
f_{u}=\sum_{i=1}^{4} a^{i i}, f_{v}=\sum_{i=1}^{4} b^{i i}
$$

$$
\Delta=c e^{f}, \quad c=\text { const. }
$$

The most general system of solutions of (2.1) is a linear transform, with constant coefficients, of any particular fundamental system of solutions $y_{k}{ }^{i}$. The equations (2.1) and (2.2) thus define four surfaces $S_{i}$, which taken in pairs determine six congruences; these in turn can be grouped in twos, and in any pair corresponding generators do not intersect. The congruences $\Gamma_{1}$ and $\Gamma_{2}$ form such a pair and the study of the correspondence existing between them by means of (2.1) and (2.2) will necessarily be a projective study.

The geometry of the correspondence between $\Gamma_{1}$ and $\Gamma_{2}$ is equivalent to the theory of the invariants and covariants of (2.1) under the most general transformation 


$$
\begin{aligned}
& y^{m}=\alpha_{m} \bar{y}^{1}+\beta_{m} \bar{y}^{2}, \quad y^{n}=\alpha_{n} \bar{y}^{3}+\beta_{n} \bar{y}^{4} \quad(m=1,2 ; n=3,4), \\
& \Delta_{12}=\alpha_{1} \beta_{2}-\alpha_{2} \beta_{1} \neq 0, \quad \Delta_{34}=\alpha_{3} \beta_{4}-\alpha_{4} \beta_{3} \neq 0,
\end{aligned}
$$

and

$$
\bar{u}=\phi(u, v), \quad \bar{v}=\psi(u, v), \quad J(\phi, \psi) \neq 0,
$$

which leaves the two congruences unaltered.* The system (2.1) under (2.4) goes over into a system of the same form whose coefficients are readily computed. It is only necessary to display eight of these, namely

$$
\begin{aligned}
& \Delta_{12} \bar{a}^{11}=\beta_{2}\left(-\alpha_{1 u}+a^{1 m} \alpha_{m}\right)-\beta_{1}\left(-\alpha_{2 u}+a^{2 m} \alpha_{m}\right), \\
& \Delta_{12} \bar{a}^{12}=\beta_{2}\left(-\beta_{1 u}+a^{1 m} \beta_{m}\right)-\beta_{1}\left(-\beta_{2 u}+a^{2 m} \beta_{m}\right), \\
& \Delta_{12} \bar{a}^{21}=-\alpha_{2}\left(-\alpha_{1 u}+a^{1 m} \alpha_{m}\right)+\alpha_{1}\left(-\alpha_{2 u}+a^{2 m} \alpha_{m}\right), \\
& \Delta_{12} \bar{a}^{22}=-\alpha_{2}\left(-\beta_{1 u}+a^{2 m} \beta_{m}\right)+\alpha_{1}\left(-\beta_{2 u}+a^{2 m} \beta_{m}\right), \\
& \Delta_{12} \bar{a}^{13}=\beta_{2} a^{1 n} \alpha_{n}-\beta_{1} a^{2 n} \alpha_{n}, \quad \Delta_{12} \bar{a}^{23}=-\alpha_{2} a^{1 n} \alpha_{n}+\alpha_{1} a^{2 n} \alpha_{n}, \\
& \Delta_{12} \bar{a}^{14}=\beta_{2} a^{1 n} \beta_{n}-\beta_{1} a^{2 n} \beta_{n}, \quad \Delta_{12} \bar{a}^{24}=-\alpha_{2} a^{1 n} \beta_{n}+\alpha_{1} a^{2 n} \beta_{n} .
\end{aligned}
$$

Another eight coefficients are secured from the substitution $(a b)(u v)$ and the remaining sixteen are obtained by applying the substitution

$$
(13)(24)(m n)
$$

to those already determined.

\section{A CANONICAL FORM FOR THE DIFFERENTIAL EQUATIONS}

New reference surfaces for the congruences may be introduced by applying (2.4) to (2.1). ' Let $\alpha_{l}, \beta_{l}$ be arbitrary pairs of solutions of the equations

$$
-\alpha_{l u}+a^{l m} \alpha_{m}=0, \quad-\beta_{l v}+b^{l m} \beta_{m}=0,
$$

so chosen that $\Delta_{12} \neq 0$. A glance at (2.6) gives the relations

$$
\bar{a}^{11}=\bar{a}^{21}=\bar{b}^{12}=\bar{b}^{22}=0,
$$

and the reference surfaces of $\Gamma_{2}$ may be chosen similarly to give the relations

$$
\bar{a}^{33}=\bar{a}^{43}=\bar{b}^{34}=\bar{b}^{44}=0 .
$$

The equations of the correspondence. now take the form (2.1) where the conditions (3.1) are satisfied. The most general transformation (2.4) and (2.5) which preserves (3.1) and leaves the reference surfaces unaltered is of the form

* The meaning here given to the letters $m$ and $n$ will be preserved throughout the discussion. The letters $l$ and $p$ will be used in the sense of $m$ and $n$ respectively. 


$$
\begin{aligned}
y^{1}=\alpha_{1}(v) \bar{y}^{1}, \quad y^{2} & =\beta_{2}(u) \bar{y}^{2}, \quad y^{3}=\alpha_{3}(v) \bar{y}^{3}, \quad y^{4}=\beta_{4}(u) \bar{y}^{4} \\
\bar{u} & =\phi(u), \quad \bar{v}=\psi(v) .
\end{aligned}
$$

\section{PAIRS OF CONGRUENCES WITH AN INTERSECTOR PROPERTY}

In his study of pairs of ruled surfaces, Lane* has shown that a family of curves, which he has called a family of intersector curves, exists on each ruled surface, the curves being defined as follows. A curve on a ruled surface $R_{1}$ is an intersector curve with respect to a second ruled surface $R_{2}$ in case the tangent at each point of the curve intersects the line $l_{2}$ which corresponds to the generator $l_{1}$ that passes through the point. We now proceed to describe a somewhat analogous situation for a pair of congruences. Let $\Gamma_{1}$ be given together with a one-parameter family of surfaces so that $l_{1}$ cuts each of the surfaces in just one point. Further let us suppose that the tangent planes to the surfaces at the points of $l_{1}$ form a pencil; then the axes of the pencils so obtained determine $\Gamma_{2}$, which, by construction, has its generators in oneto-one correspondence with the generators $\dagger$ of $\Gamma_{1}$. The surfaces of the family will be called intersector surfaces of $\Gamma_{1}$ with respect to $\Gamma_{2}$, and we shall say that $\Gamma_{1}$ possesses property $I$ with respect to $\Gamma_{2}$.

The conditions under which the reference surfaces of $\Gamma_{1}$ can be chosen to have the intersector property with respect to $\Gamma_{2}$ are readily determined. For convenience, let $\alpha_{1}=-\lambda_{1} \alpha_{2}, \beta_{1}=-\lambda_{2} \beta_{2}$, where $\lambda_{1} \neq \lambda_{2}$, since $\Delta_{12} \neq 0$. Then $\lambda_{1}$ and $\lambda_{2}$ appear as distinct solutions of the equations

$$
\begin{aligned}
& \lambda_{u}=a^{21} \lambda^{2}+\left(a^{11}-a^{22}\right) \lambda-a^{12}, \\
& \lambda_{v}=b^{21} \lambda^{2}+\left(b^{11}-b^{22}\right) \lambda-b^{12},
\end{aligned}
$$

which can have analytic solutions only when the equation

$$
p \lambda^{2}+q \lambda+r=0, \quad D^{2}=q^{2}-4 p r,
$$

is satisfied, where

$$
\begin{aligned}
p & =a_{v}^{21}-b_{u}{ }^{21}+a^{2 m} b^{m 1}-b^{2 m} a^{m 1}, \\
(4.3) & q=a_{v}^{11}-b_{u}^{11}-\left(a_{v}^{22}-b_{u}^{22}\right)+a^{1 m} b^{m 1}-b^{1 m} a^{m 1}-\left(a^{2 m} b^{m 2}-b^{2 m} a^{m 2}\right), \\
r & =a_{v}^{12}-b_{u}^{12}+a^{1 m} b^{m 2}-b^{1 m} a^{m 2} .
\end{aligned}
$$

* E. P. Lane, Ruled surfaces with generators in one-to-one correspondence, these Transactions, vol. 25 (1923), pp. 287-290.

$\dagger$ Correspondences of this type have been discussed by G. Fubini, Su alcune classi di congruenze di rette e sulle transformazione delle superficie $R$, Annali di Matematica, (4), vol. 1 (1923-24), pp. 241-257. 
The expressions for $p, q, r$ may be written as

$$
\begin{aligned}
& p=-a^{2 n} b^{n 1}+b^{2 n} a^{n 1} \\
& q=-\left(a^{1 n} b^{n 1}-b^{1 n} a^{n 1}\right)+\left(a^{2 n} b^{n 2}-b^{2 n} a^{n 2}\right) \\
& r=-a^{1 n} b^{n 2}+b^{1 n} a^{n 2}
\end{aligned}
$$

by virtue of the conditions (2.2).

In case $p \equiv q \equiv r \equiv 0$, the pair of equations (4.1) will be spoken of as a Riccati system of differential equations, with the integrability conditions

$$
\begin{aligned}
a^{1 n} b^{n 2} & =b^{1 n} a^{n 2}, \\
a^{1 n} b^{n 1}-a^{2 n} b^{n 2} & =b^{1 n} a^{n 1}-b^{2 n} a^{n 2}, \\
a^{2 n} b^{n 1} & =b^{2 n} a^{n 1} .
\end{aligned}
$$

The conditions (4.5) are necessary and sufficient for the existence of a one-parameter set of solutions $\lambda$ of the Riccati system (4.1), hence the theorem. The conditions (4.5) on the coefficients of (2.1) provide a necessary and sufficient condition that $\Gamma_{1}$ possess property $I$ with respect to $\Gamma_{2}$. Furthermore, the intersector surfaces of $\Gamma_{1}$ with respect to $\Gamma_{2}$ are determined by solving a Riccati system.

The intersector surfaces are in fact defined by

$$
x=y^{1}+\lambda y^{2},
$$

where $\lambda=\lambda(u, v)$ is an arbitrary solution of (4.1).

Necessary and sufficient conditions that $\Gamma_{2}$ possess property $I$ with respect to $\Gamma_{1}$ are obtained by applying (2.7) to (4.5). These conditions are not a consequence of (4.5), so that the two sets of conditions taken together provide a necessary and sufficient condition that $\Gamma_{1}$ and $\Gamma_{2}$ possess property $I$ with respect to each other.

There remain for discussion the solutions of (4.1) when not all of $p, q, r$ vanish identically. In this case the analytic solutions of (4.1) are not more than two in number; for, if $\lambda=\lambda(u, v)$ is an analytic solution of (4.1) it is thereby a solution of (4.2). Suppose conversely that $\lambda$ is a solution of (4.2); the conditions under which $\lambda$ is a solution of (4.1) are the following:

$$
\begin{aligned}
& p q_{u}-p_{u} q=2 p\left(a^{12} p+a^{21} r\right)+q\left(-a^{21} q+\left(a^{11}-a^{22}\right) p\right), \\
& p r_{u}-p_{u} r=q\left(a^{12} p+a^{21} r\right)+2 r\left(-a^{21} q+\left(a^{11}-a^{22}\right) p\right), \\
& p q_{v}-p_{v} q=2 p\left(b^{12} p+b^{21} r\right)+q\left(-b^{21} q+\left(b^{11}-b^{22}\right) p\right), \\
& p r_{v}-p_{v} r=q\left(b^{12} p+b^{21} r\right)+2 r\left(-b^{21} q+\left(b^{11}-b^{22}\right) p\right) .
\end{aligned}
$$

In case $D \neq 0$ the conditions (4.7) are necessary and sufficient for the existence 
of two and only two surfaces associated with $\Gamma_{1}$ having the intersector property with respect to $\Gamma_{2}$.

The two surfaces are given by (4.6) where $\lambda$ satisfies (4.2). They may be chosen as reference surfaces for $\Gamma_{1}$ in which case we have the relations

$$
a^{12}=a^{21}=b^{12}=b^{21}=0 .
$$

When $D=.0$ the conditions (4.7) reduce to the form

$$
\left|\begin{array}{l}
p q_{u}-p_{u} q, \quad p r_{u}-p_{u} r \\
p q_{v}-p_{v} q, \quad p r_{v}-p_{v} r
\end{array}\right|=0 .
$$

In case $D=0$, and $p, q, r$ are not all identically zero, (4.9) provides a necessary and sufficient condition for the existence of one and only one surface associated with $\Gamma_{1}$ having the intersector property with respect to $\Gamma_{2}$. As before, the surface in question is given by (4.6) and (4.2). The surface may be chosen as the reference surface $S_{1}$ for $\Gamma_{1}$ in which case $a^{12}=b^{12}=0$.

\section{The THEORY OF A RicCATI SYSTEM OF DIFFERENTIAL EQUATIONS}

Let there be given a Riccati system

$$
\begin{aligned}
& \lambda_{u}=a_{1} \lambda^{2}+a_{2} \lambda+a_{3}, \\
& \lambda_{v}=b_{1} \lambda^{2}+b_{2} \lambda+b_{3},
\end{aligned}
$$

where $a_{r}, b_{r}(r=1,2,3)$ are analytic functions of $u$ and $v$, with the integrability conditions

$$
\begin{aligned}
a_{1 v}-b_{1 u}+\left(a_{1} b_{2}-a_{2} b_{1}\right) & =0 \\
a_{2 v}-b_{2 u}+2\left(a_{1} b_{3}-a_{3} b_{1}\right) & =0 \\
a_{3 v}-b_{3 u}+\left(a_{2} b_{3}-a_{3} b_{2}\right) & =0
\end{aligned}
$$

Theorems analogous to those for an ordinary Riccati equation* may be obtained for a Riccati system (5.1). Thus, for instance, the cross ratio of any four analytic solutions of a Riccati system is constant.

Let us now return to the geometrical considerations from which arose the discussion of the Riccati system of equations. In the first place the meaning of the term intersector surface becomes apparent from the following theorem. When $\Gamma_{1}$ possesses property $I$ with respect to $\Gamma_{2}$, the intersector curves on all the ruled surfaces $R_{1}$ of $\Gamma_{1}$ with respect to the corresponding ruled surfaces $R_{2}$ of $\Gamma_{2}$ form themselves into the intersector surfaces of $\Gamma_{1}$ with respect to $\Gamma_{2}$. This theorem can be phrased more directly by saying that any ruled surface

* For a discussion of this equation see L. P. Eisenhart, Differential Geometry, Boston, Ginn, 1909, pp. 25-26. 
$R_{1}$ of $\Gamma_{1}$ meets the one-parameter family of intersector surfaces along its intersector curves with respect to the corresponding ruled surface $R_{2}$ of $\Gamma_{2}$. In this form the theorem is geometrically evident and we can in fact exhibit the Riccati equation defining the intersector curves of $R_{1}$. The parameters $u$ and $v$ can always be chosen so that any $R_{1}$ of $\Gamma_{1}$ is given by $v=v(u)$ which also defines $R_{2}$. We have from (2.1) the relations

$$
\frac{d y^{i}}{d u}=\left(a^{i j}+v^{\prime} b^{i j}\right) y^{j}
$$

which, on comparison with Lane, loc. cit., p. 282, (1), are the equations for the correspondence between $R_{1}$ and $R_{2}$, and on the other hand,

$$
\frac{d \lambda}{d u}=\left(a^{21}+v^{\prime} b^{21}\right) \lambda^{2}+\left\{\left(a^{11}-a^{22}\right)+v^{\prime}\left(b^{11}-b^{22}\right)\right\} \lambda-\left(a^{12}+v^{\prime} b^{12}\right),
$$

which, from (5.3) and Lane, loc. cit., p. 288, (13), is the required Riccati equation.

The cross ratio of any four solutions of a Riccati system having been shown to be constant, we have proved that when $\Gamma_{1}$ possesses property $I$ with respect to $\Gamma_{2}$, any four intersector surfaces cut each generator $l_{1}$ of $\Gamma_{1}$ in a set of four points which have the same cross ratio on all generators of $\Gamma_{1}$. This theorem is a generalization of the theorem proved by Lane, loc. cit., p. 289, for intersector curves.

Since the theory of a Riccati equation is equivalent to the theory of the intersector curves on a given ruled surface, with respect to some associated ruled surface, ${ }^{*}$ it is natural to inquire if there is any sense in which it can be said that the theory of a Riccati system is equivalent to the theory of the intersector surfaces of a given congruence which possesses property $I$ with respect to some associated congruence. The answer is in the negative, for let us assume that we are given a Riccati system (5.1) and a congruence $\Gamma_{1}$; then it is not possible to determine a second congruence $\Gamma_{2}$ with respect to which $\Gamma_{1}$ possesses property $I$, and at the same time have the intersector surfaces defined by (5.1). We omit the proof of this statement.

If the parametric ruled surfaces of the congruence $\Gamma_{1}$ for the correspondence (2.1) are chosen as the developables of $\Gamma_{1}$ the relations

$$
a^{13} a^{24}-a^{14} a^{23}=0, \quad b^{13} b^{24}-b^{14} b^{23}=0
$$

are obtained, evidently necessary and sufficient conditions that the developables of $\Gamma_{1}$ be the parametric surfaces for that congruence in the corre-

* Lane, loc. cit., p. 289. 
pondence defined by (2.1). The application of the substitution (13) (24) to (5.4) yields the relations

$$
a^{31} a^{42}-a^{32} a^{41}=0, \quad b^{31} b^{42}-b^{32} b^{41}=0
$$

which are necessary and sufficient that the developables of $\Gamma_{2}$ be the parametric surfaces for $\Gamma_{2}$.

\section{The INTERSECtor SURFACES}

When $\Gamma_{1}$ possesses property $I$ with respect to $\Gamma_{2}$ the equations of the correspondence and of the intersector surfaces reduce to simpler forms. The intersector surfaces form a family, and the points of any pair of the surfaces are in correspondence, where corresponding points lie on a generator $l_{1}$ of $\Gamma_{1}$. The consideration of corresponding nets of curves on these surfaces suggests itself. When the nets of curves are conjugate nets two distinct possibilities occur. The first of these, in which case the developables of $\Gamma_{1}$ trace out conjugate nets on all the intersector surfaces, has been studied by Fubini* who at the same time recognized $\dagger$ its relation to certain theorems of Darboux. $\neq$ This relation may be fully established by means of the present theory. The second case occurs when each of the congruences $\Gamma_{1}$ and $\Gamma_{2}$ possesses property $I$ with respect to the other.

When $\Gamma_{1}$ possesses property $I$ with respect to $\Gamma_{2}$ the conditions (4.5) are satisfied, and by choosing an arbitrary pair of intersector surfaces as reference surfaces for $\Gamma_{1}$, the coefficients of (2.1) can be made to satisfy the additional relations

$$
a^{11}=a^{12}=a^{21}=a^{22}=b^{12}=b^{21}=0, b^{11}=b^{22} .
$$

The intersector surfaces are then given by (4.6), where $\lambda=$ const. By means of (4.6) the coefficients $D, D^{\prime}, D^{\prime \prime}$ may be computed for an arbitrary intersector surface $S_{x}$; they are

where

$$
\begin{aligned}
D & =\left(a^{1 n} a^{n 2}+\lambda\left(a^{2 n} a^{n 2}-a^{1 n} a^{n 1}\right)-\lambda^{2} a^{2 n} a^{n 1}\right) F \Delta, \\
D^{\prime} & =\left(a^{1 n} b^{n 2}+\lambda\left(a^{2 n} b^{n 2}-a^{1 n} b^{n 1}\right)-\lambda^{2} a^{2 n} b^{n 1}\right) F \Delta, \\
D^{\prime \prime} & =\left(b^{1 n} b^{n 2}+\lambda\left(b^{2 n} b^{n 2}-b^{1 n} b^{n 1}\right)-\lambda^{2} b^{2 n} b^{n 1}\right) F \Delta,
\end{aligned}
$$

$$
F=\left|\begin{array}{ll}
a^{13}+\lambda a^{23}, & a^{14}+\lambda a^{24} \\
b^{13}+\lambda b^{23}, & b^{14}+\lambda b^{24}
\end{array}\right|
$$

* Fubini, loc. cit., pp. 246-250.

$\dagger$ Ibid., p. 242.

‡ G. Darboux, Leçons sur la Théorie Générale des Surfaces, 1915, 2e édition, livre IV, chap. X, pp. 241-252. 
When $F=0$, (4.6) defines the focal surfaces of $\Gamma_{1}$. Evidently a focal surface cannot have the intersector property; the focal surfaces must also be distinct, otherwise the intersector surfaces degenerate into intersector curves, a situation which will be excluded.

The equations (6.2) show that the parametric surfaces of $\Gamma_{1}$ will trace out a conjugate net of curves on every intersector surface when the following conditions are satisfied:

$$
a^{1 n} b^{n 2}=0, \quad a^{2 n} b^{n 2}=a^{1 n} b^{n 1}, \quad a^{2 n} b^{n 1}=0 ；
$$

from (4.5) the further relations

$$
b^{1 n} a^{n 2}=0, \quad b^{2 n} a^{n 2}=b^{1 n} a^{n 1}, \quad b^{2 n} a^{n 1}=0
$$

are obtained. The conditions $\left(6.4_{1}\right),\left(6.4_{3}\right)$ and $\left(6.5_{1}\right),\left(6.5_{3}\right)$ result in the equations

$$
a^{2 n} b^{n 2}=\frac{a^{24} b^{42}}{a^{13} b^{31}} a^{1 n} b^{n 1}, \quad b^{2 n} a^{n 2}=\frac{b^{24} a^{42}}{b^{13} a^{31}} b^{1 n} a^{n 1} .
$$

A comparison of these two relations with $\left(6.4_{2}\right)$ and $\left(6.5_{2}\right)$ leads to three possible consequences of (6.4) and (6.5), namely:

$$
\begin{aligned}
a^{1 n} b^{n 1} & =0, & b^{1 n} a^{n 1} & =0, \\
a^{24} b^{42}-a^{13} b^{31} & =0, & b^{24} a^{42}-b^{13} a^{31} & =0,
\end{aligned}
$$

and

$$
a^{1 n} b^{n 1}=0, \quad b^{24} a^{42}-b^{13} a^{31}=0,
$$

or the alternatives

$$
b^{1 n} a^{n 1}=0, \quad a^{24} b^{42}-a^{13} b^{31}=0,
$$

which are not essentially different from (6.8).

We turn attention to the situation corresponding to (6.4), (6.5), (6.6). These conditions taken together may be written in the form

$$
a^{l n} b^{n m}=0, \quad b^{l n} a^{n m}=0 .
$$

As a consequence we note first of all that (5.4) and (5.5) are evidently satisfied. Geometrically, (6.10) then implies that the developables of $\Gamma_{1}$ are parametric, and the corresponding parametric surfaces for $\Gamma_{2}$ are the developables of that congruence. The developables of the pair of congruences thus correspond, and those of $\Gamma_{1}$ trace out a conjugate net of curves on every intersector surface. Secondly, we see on comparing (2.2) and (6.1) with (6.10) that

$$
b_{u}^{11}=0 \text {. }
$$


In case $\Gamma_{1}$ possesses property $I$ with respect to $\Gamma_{2}$ with the further property that the developables of $\Gamma_{1}$ trace out a conjugate net of curves on every intersector surface, we shall say for brevity that $\Gamma_{1}$ possesses property $I_{1}$ with respect to $\Gamma_{2}$.

The following two theorems concerning necessary and sufficient conditions for property $I_{1}$ are stated without proof.

Let $\Gamma_{1}$ possess property $I$ with respect to $\Gamma_{2}$ and write the equations of the correspondence in the form (2.1), (6.1). The equation (6.11) is a necessary and sufficient condition that $\Gamma_{1}$ possess property $I_{1}$ with respect to $\Gamma_{2}$.

When the equations of the correspondence between $\Gamma_{1}$ and $\Gamma_{2}$ are in general form (2.1), the conditions

$$
a^{l n} b^{n m}=b^{l n} a^{n m}
$$

are necessary and sufficient that $\Gamma_{1}$ possess property $I_{1}$ with respect to $\Gamma_{2}$. In case the developables of $\Gamma_{1}$ are the parametric surfaces of the congruence these conditions take the form (6.10).

As a consequence of these results two theorems due to Fubini $†$ may be proved without difficulty:

When $\Gamma_{1}$ possesses property $I$ with respect to $\Gamma_{2}$ the developables of $\Gamma_{1}$ cannot trace out a conjugate net on any of the intersector surfaces unless $\Gamma_{1}$ possess property $I_{1}$ with respect to $\Gamma_{2}$.

When $\Gamma_{1}$ possesses property $I_{1}$ with respect to $\Gamma_{2}$, the developables of the congruences correspond.

We are now in a position to connect up the fundamental theorem of Darboux $\ddagger$ with the present theory. Let two surfaces $S_{1}$ and $S_{2}$ be given in one-to-one point correspondence. The lines joining corresponding points generate a congruence $\Gamma_{1}$, while the lines of intersection of the tangent planes to the surfaces at corresponding points generate a second congruence $\Gamma_{2}$. Corresponding generators do not intersect unless $S_{1}$ and $S_{2}$ are the focal surfaces of $\Gamma_{1}$, in which case $l_{1}$ and $l_{2}$ coincide. This case is excluded from the discussion.

When the developables of $\Gamma_{1}$ trace out conjugate nets on each of the surfaces $S_{1}$ and $S_{2}$ the surfaces are said by some writers to be in the relation of a transformation $F$, or briefly in relation $F . \S$

* This correspondence was first studied by Fubini, loc. cit., pp. 246-250.

$\dagger$ Ibid., pp. 244 and 246.

$\ddagger$ Darboux, loc. cit., p. 244.

\&. P. Eisenhart, Transformations of Surfaces, Princeton University Press, 1923, p. 34. 
We can now prove the following theorem:

$S_{1}$ and $S_{2}$ are in relation $F$ if and only if $\Gamma_{1}$ possesses property $I_{1}$ with respect to $\Gamma_{2}$.

To show this we note to begin with that (4.7) is necessarily satisfied. As a consequence, the additional relations

$$
a^{11}=a^{12}=a^{21}=b^{12}=b^{21}=b^{22}=0
$$

can be made to hold. Further, the developables of $\Gamma_{1}$ may be chosen as the parametric surfaces of the congruence, so that the conditions (5.4) are satisfied. $S_{1}$ and $S_{2}$ are now the reference surfaces for $\Gamma_{1}$, and the developables of $\Gamma_{1}$ will trace out a conjugate net of curves on each side of these surfaces only when

$$
a^{1 n} b^{n 2}=0, \quad a^{2 n} b^{n 1}=0,
$$

$S_{1}$ and $S_{2}$ being non-degenerate. The conditions (2.2) give the further relations

$$
b^{1 n} a^{n 2}=0, \quad b^{2 n} a^{n 1}=0 .
$$

The equations (5.4) may now be brought into play and (6.10) follows thus showing that $\Gamma_{1}$ possesses property $I_{1}$ with respect to $\Gamma_{2}$.

Conversely, if $\Gamma_{1}$ possesses property $I_{1}$ with respect to $\Gamma_{2}, S_{1}$ and $S_{2}$ are evidently intersector surfaces and the developables of $\Gamma_{1}$ trace out a conjugate net of curves on each of the surfaces.

Let us turn next to the consideration of (6.4), (6.5) and (6.7). The conditions found from (4.5) by use of (2.7) can be shown to be satisfied so that $\Gamma_{2}$ necessarily possesses property $I$ with respect to $\Gamma_{1}$, and the parametric surfaces of $\Gamma_{2}$ trace out a conjugate net of curves on every intersector surface of $\Gamma_{2}$ with respect to $\Gamma_{1}$.

Suppose now, conversely, we assume that each of $\Gamma_{1}, \Gamma_{2}$ possesses property $I$ with respect to the other. The equations of the correspondence can be written in the form (2.1), with (6.1) and with the additional conditions

$$
b^{n p}=0, \quad a^{33}=a^{44}, \quad a^{34}=a^{43}=0 .
$$

We note also from (2.3) that the equation

$$
a_{v}^{33}=b_{u}^{11}
$$

is satisfied. The coefficients of this reduced system of equations satisfy (4.5), and the conditions found from (4.5) and (2.7) by the nature of the correspondence which they represent.

Suppose further that the coefficients of (2.1) satisfy (6.7). The con- 
ditions (6.7) are equivalent to a particular choice of the parametric ruled surfaces for the correspondence. Then it may be shown that (6.4), (6.5) hold provided the condition

$$
a^{24} a^{42}-a^{13} a^{31} \neq 0
$$

be maintained.

We have thus proved the following theorem.

When each of $\Gamma_{1}, \Gamma_{2}$ possesses property I with respect to the other there exists ordinarily one and just one net of ruled surfaces for each congruence which traces out a conjugate net of curves on every intersector.surface associated with that congruence.

The third possibility for consideration is expressed by (6.4), (6.5), (6.8). We note that (6.8) is made up of $\left(6.6_{1}\right)$ and $\left(6.7_{2}\right)$. We might expect interaction between $\left(6.6_{1}\right)$ and $\left(6.7_{2}\right)$, creating conditions not already found in the discussion of the previous cases; the nature of $\left(6.6_{1}\right)$ and $\left(6.7_{2}\right)$, however, shows that this is not possible. Hence we need not expect a third type of relation between $\Gamma_{1}$ and $\Gamma_{2}$ for which conjugate nets of curves on the intersector surfaces of $\Gamma_{1}$ with respect to $\Gamma_{2}$ are in correspondence.

If the parametric surfaces of $\Gamma_{1}$ are to trace out an asymptotic net of curves on every intersector surface of $\Gamma_{1}$ with respect to $\Gamma_{2}$, three possibilities are secured as in the discussion for conjugate nets of curves.

The conditions secured in the first case cannot hold, for they imply that the developables are the parametric surfaces for $\Gamma_{1}$, and Fubini* has shown that the developables of $\Gamma_{1}$ cannot trace out the asymptotic nets on all the intersector surfaces.

In the second case the relations

$$
\begin{aligned}
& a^{4 m} a^{m 4}=a^{3 m} a^{m 3}, \quad a^{n m} a^{m p}=0, \\
& b^{4 m} b^{m 4}=b^{3 m} b^{m 3}, \quad b^{n m} b^{m p}=0 \quad(n \neq p)
\end{aligned}
$$

may be obtained in a manner entirely analogous to the discussion of the equations (6.4), (6.5), (6.7). We are unable to say anything further about this case, other than that it does not appear likely to yield conditions suffcient for the desired correspondence of the asymptotic nets on the intersector surfaces.

\section{The tRansformation of LAPLACE}

When $\Gamma_{1}$ possesses property $I_{1}$ with respect to $\Gamma_{2}$, the system (2.1) may be simplified so that the relations

* Fubini, loc. cit., p. 246. 


$$
a^{l m}=b^{l m}=0
$$

are satisfied.

Let the developables of $\Gamma_{1}$ be chosen as the parametric surfaces for the congruence. The equations (5.4) and (5.5) are satisfied since the developables of the congruences correspond; and (6.12) becomes (6.10). From (5.4) and (7.1) we have the equations

$$
y_{u}^{1}=t_{1} y_{u}^{2}, \quad y_{v}^{1}=t_{2} y_{v}^{2},
$$

where

$$
t_{1}=a^{13} / a^{23}=a^{14} / a^{24}, t_{2}=b^{13} / b^{23}=b^{14} / b^{24}, t_{1} \neq t_{2} .
$$

A generator $l_{1}$ of $\Gamma_{1}$ is cut by an arbitrary intersector surface in the point $P_{x}$ whose coördinates are given by (4.6) with $\lambda=$ const. From (4.6) and (7.2) we find

$$
x_{u}=\left(t_{1}+\lambda\right) y_{u^{2}}{ }^{2} \quad x_{v}=\left(t_{2}+\lambda\right) y_{v}{ }^{2},
$$

and certain theorems concerning the nature of the correspondence, proved by Fubini* and Darboux, $\uparrow$ now follow without difficulty.

Since the developables of $\Gamma_{1}$ meet any intersector surface in a conjugate net of curves, these curves form the parametric net for the surface, where it is assumed that (5.4) and (5.5) are in force, and the Laplace equation. for the surface may be determined as

$$
x_{u v}=\frac{t_{2}+\lambda}{t_{1}+\lambda} b x_{u}+\frac{t_{1}+\lambda}{t_{2}+\lambda} c x_{v},
$$

where

$$
b=\frac{-t_{1 v}}{t_{1}-t_{2}}, c=\frac{t_{2 u}}{t_{1}-t_{2}} .
$$

In case $b=0$ or $c=0$, one or the other of the focal surfaces of $\Gamma_{2}$ degenerates into a curve, so that we may assume $b c \neq 0$.

The following theorem may now be stated without proof.

The locus of the ray-points $P_{\sigma}$ of the u-curves of the intersector surfaces at the points of a generator $l_{1}$ of $\Gamma_{1}$ is the v-tangent to the focal surface for the $u$-developables at the focal point of $l_{1}$.

The focal point of $l_{1}$ is a singular point for the locus.

A corresponding theorem may be stated by interchanging $u$ and $v$, and replacing $\sigma$ by $\rho$.

* Fubini, loc. cit., pp. 246 and 247.

$\dagger$ Darboux, loc. cit., p. 245. The theorem of Darboux appears as a special case of the theorem of Fubini, loc. cit., p. 247, by virtue of the theorem which has been proved in $\S 6$. 
Suppose now we fix upon a particular intersector surface $x=y^{1}+\lambda_{0} y^{2}$, where $\lambda=\lambda_{0}$ is some constant. For this surface we find

$$
x_{u}=\left(t_{1}+\lambda_{0}\right) y_{u}{ }^{2}, \quad t_{1}=t_{1}(u, v) .
$$

The equation $t_{1}(u, v)+\lambda_{0}=0$ defines a certain curve on the surface and $x_{u}=0$ all along this curve. Every point of this curve is a point $P_{r^{1}}$, its coordinates having the form $\tau^{1}=y^{1}-t_{1} y^{2}$, hence the curve also lies on the focal surface of the $u$-developable of $\Gamma_{1}$. At the same time we find

$$
x_{v}=\left(t_{2}+\lambda_{0}\right) y_{v}{ }^{2}, \quad t_{2}=t_{2}(u, v),
$$

so that $x_{v}=0$ all along the curve defined by $t_{2}(u, v)+\lambda_{0}=0$, which is the intersection of the given surface and the focal surface for the $v$-developables of $\Gamma_{1}$. The following theorem has thus been proved.

When $\Gamma_{1}$ possesses property $I_{1}$ with respect to $\Gamma_{2}$ every intersector surface of $\Gamma_{1}$ with respect to $\Gamma_{2}$ contains two singular curves which are the intersections of the surface with the focal surfaces of $\Gamma_{1}$.

The ray-points for the reference surface $S_{1}$ at $P_{y^{1}}$ may be written as $P_{\rho^{1}}, P_{\sigma^{1}}$, and likewise the ray-points of $S_{2}$ at $P_{y^{2}}$ as $P_{\rho^{2}}, P_{\sigma^{2}}$ respectively. The line $l_{\rho^{1} \rho^{2}}$ is the locus of the ray-point $P_{\rho}$ and $l_{\sigma^{1} \sigma^{2}}$ is the locus of the ray-point $P_{\sigma}$. Two lines have thus been determined which correspond to $l_{1}$, thus generating the pair of congruences $\Gamma_{\rho}, \Gamma_{\sigma}$.

The Laplace transforms of $\Gamma_{1}$ are defined by means of the focal surfaces of $\Gamma_{1}$, whose equations are

$$
\tau^{1}=y^{1}-t_{1} y^{2}, \tau^{2}=y^{1}-t_{2} y^{2},
$$

respectively. The congruence $\Gamma_{\tau^{2} \tau_{v}^{2}}$ is the congruence $\Gamma_{1}$, but $\Gamma_{\tau 2 \tau_{u}^{2}}$ which, by definition, is called the first Laplace transform of $\Gamma_{1}$, has been shown to be $\Gamma_{\rho}$. The congruence $\Gamma_{\tau 1 \tau_{u}^{1}}$ is again $\Gamma_{1}$, but $\Gamma_{\tau 1 r_{v}^{1}}$ which, by definition, is the minus first Laplace transform of $\Gamma_{1}$, is $\Gamma_{\sigma}$.

The points $P_{y_{k}^{1}}, P_{y_{v}^{1}}$ are the focal points for $l_{2}$, and $\Gamma_{y_{u}^{1} \nu_{u v}^{1}}$ is $\Gamma_{2}$ while $\Gamma_{\nu_{u}^{1} y_{u u}^{1}}^{1}$ is the first Laplace transform of $\Gamma_{2} . \Gamma_{y_{v}^{1} y_{u v}^{1}}$ is again $\Gamma_{2}$, while $\Gamma_{\nu_{v}^{1} y_{v v}^{1}}$ is the minus first Laplace transform of $\Gamma_{2}$.

The equations (2.1) may be set up for the correspondence between the first Laplace transforms of $\Gamma_{1}$ and $\Gamma_{2}$ and the theorem follows:

When $\Gamma_{1}$ possesses property $I_{1}$ with respect to $\Gamma_{2}$, the first Laplace transform of $\Gamma_{1}$ possesses property $I_{1}$ with respect to the first Laplace transform of $\Gamma_{2}{ }^{*}$ The intersector surfaces for the derived correspondence are the first Laplace transforms of the intersector surfaces for the given correspondence.

* Fubini, loc. cit., p. 249. 
The corresponding statement can be made for the minus first Laplace transforms.

\section{RECIPROCAL CONGRUENCES}

The Green reciprocal relation for a pair of congruences represents a particular type of the correspondence defined by (2.1). As a conclusion to the present discussion the equations of the correspondence between an arbitrary pair of reciprocal congruences may be exhibited.

Let the four homogeneous coördinates $x_{k}$ of an arbitrary point $P_{x}$ on a surface $S_{x}$ be given as analytic functions of two independent variables $u$ and $v$. Suppose further that $S_{x}$ is non-degenerate and non-developable, and is referred to its asymptotic net. Then the functions $x_{k}$ are a fundamental set of solutions of a completely integrable system of partial differential equations which may be reduced to the Fubini form

$$
x_{u u}=p x+\theta_{u} x_{u}+\beta x_{v}, \quad x_{v v}=q x+\gamma x_{u}+\theta_{v} x_{v},
$$

where $\theta=\log \beta \gamma$, and where certain integrability conditions are satisfied.

The points $P_{\rho}, P_{\sigma}$ defined by

$$
\rho=x_{u}+b x, \quad \sigma=x_{v}+a x,
$$

where $a$ and $b$ are functions of $u$ and $v$, are evidently points which lie in the tangent plane of $S_{x}$ at $P_{x}$. The reciprocal of $l_{\rho \sigma}$ in the osculating quadric of $S_{x}$ at $P_{x}$ is the line $l_{x y}$ which joins $P_{x}$ and $P_{y}$, the latter being defined by

$$
y=a x_{u}+b x_{v}+x_{u v} .
$$

Two congruences $\Gamma_{x y}, \Gamma_{\rho \sigma}$ have thus been determined in one-to-one correspondence, so that corresponding generators do not intersect. The coefficients $a^{i j}$ of the system (2.1) for the correspondence are as follows:

$$
\begin{array}{llll}
a^{11}=-b, & a^{12}=0, & a^{13}=1, & a^{14}=0, \\
a^{21}=P, & a^{22}=\theta_{u}+b, & a^{23}=A, & a^{24}=F^{\prime}, \\
a^{31}=F, & a^{32}=0, & a^{33}=\theta_{u}+b, & a^{34}=\beta, \\
a^{41}=a_{u}+a b, & a^{42}=1, & a^{43}=0, & a^{44}=-b,
\end{array}
$$

where we have placed

$$
\begin{aligned}
A & =\theta_{u v}+a_{u}-a b+\beta \gamma, \\
F & =p-b \theta_{u}-\beta a+b_{u}-b^{2}, \\
F^{\prime} & =F+2 a \beta+\beta \theta_{v}, \\
P & =a\left(p-F^{\prime}\right)-A b+p_{v}+\beta q .
\end{aligned}
$$


A. J. COOK

The coefficients $b^{i j}$ of (2.1) may be found from (8.4) and (8.5) by means of the substitution

$$
\left(\begin{array}{llllllll}
a & \beta & u & 3 & A & F & F^{\prime} & P \\
b & \gamma & v & 4 & B & G & G^{\prime} & Q
\end{array}\right)
$$

The coefficients of (5.3) taken in conjunction with (8.4) and (8.6) are in substance those given by Lane* for his theory of pairs of reciprocal ruled surfaces.

* Lane, loc. cit., p. 287. Lane here uses the Wilczynski form of the equations for $S_{x}$.

UNIVERSTTY OF Alberta,

Edmonton, Canada 